\title{
Value Tools for Patients in Cancer Care
}

\author{
Katy Winckworth-Prejsnar, MPH${ }^{\mathrm{a}}$; Lisa Korin Lentz, $\mathrm{MPH}^{\mathrm{a}}$; Elizabeth A. Nardi, $\mathrm{MS}^{\mathrm{a}}$; Sandhya Pruthi, MD \\ C. Lyn Fitzgerald, MJa; and Robert W. Carlson, MD ${ }^{\mathrm{a}, \mathrm{c}}$
}

\section{Abstract}

In order to empower patients as partners in their healthcare decisions, there is an identified need for value tools that provide enough information to help them make decisions regarding their cancer care journey. NCCN convened a multistakeholder working group to identify the gaps and needs of current value tools and develop a set of findings and recommendations for the evolution of value tools for patients. The findings and recommendations of the working group were then presented at the Value Tools for Patients in Cancer Care Patient Advocacy Summit in December 2016, and multistakeholder roundtable panel discussions explored these findings and recommendations along with additional items. This article encapsulates the discussion from the NCCN Working Group meetings and the NCCN Patient Advocacy Summit, including identified gaps and needs in defining value in cancer care, identified principles and parameters of value tools for patients in cancer care, and consensus statements and recommendations offered by the NCCN Working Group.

J Natl Compr Canc Netw 2017;15(7):872-877 doi:10.6004/jncen.2017.0118

\section{Executive Summary}

The mission to define and deliver high-value cancer care is a key focus across the oncology community and will continue to drive health reform efforts. Healthcare reform calls for a shift from fee-for-service payment to new value-based payment models, often incentivizing the incorporation of the patient experience. Patients have become more active in their role of defining value; however, there are varying definitions of value among stakeholders. Patients, for example, may view high-value care as any combination of trust, transparency, and effective communication with providers; care coordination; survivorship care; quality of life (QOL); toxicity management; limited travel for care; and limited financial risk. To this end, patient care is highly individualized.

To empower patients as partners in their healthcare decisions, there is an identified need for tools that provide information to help patients make decisions regarding their individual cancer care journey. Many oncology organizations have recently initiated efforts to develop

From anational Comprehensive Cancer Network, Fort Washington,

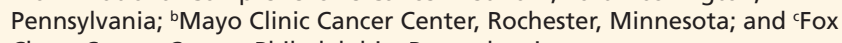
Chase Cancer Center, Philadelphia, Pennsylvania.

Submitted May 26, 2017; accepted for publication June 6, 2017.

The authors have disclosed that they have no financial interests, value tools for various audiences, including healthcare professionals, payers, and patients. These tools include, but are not limited to, NCCN Clinical Practice Guidelines in Oncology (NCCN Guidelines) with NCCN Evidence Blocks, ASCO Value Framework, COmprehensive Score for financial Toxicity (COST) tool, Consumer-Based Cancer Care Value Index, PatientCentered Value Model Rubric, the Mayo Clinic Breast Cancer Surgical Decision Support mobile device tool, and the Cancer Experience Registry. It is important to understand what value tools should look like and, importantly, how patient-oriented value tools can interface with those designed for medical professionals, how they would synergize, and where gaps exist.

To discuss the current value tools for patients in cancer care, NCCN convened a multistakeholder working group comprised of patients, patient advocacy organizations, providers, industry, pharmacists, financial counselors, social workers, and health services researchers (see supplemental eAppendix 1, available with this article at

arrangements, affiliations, or commercial interests with the manufacturers of any products discussed in this article or their competitors.

Correspondence: Katy Winckworth-Prejsnar, MPH, NCCN, 275 Commerce

Drive, Suite 300, Fort Washington, PA 19034.

E-mail: winckworthprejsnar@nccn.org 
INCCN.org). The NCCN Value Tools for Patients in Cancer Care Working Group was charged with identifying the gaps and needs of current value tools and developing a set of findings and recommendations for the evolution of tools that include the definition of a value tool for patients, principles and parameters, and a measurement of utility and validation.

The working group convened in August 2016, with subsequent teleconferences. In addition, NCCN held the NCCN Patient Advocacy Summit: Value Tools for Patients on December 9, 2016, in Washington, DC, which included additional thought leaders representing the aforementioned groups and relevant stakeholders (supplemental eAppendix 2). This article encapsulates the discussion from the NCCN Working Group meetings and the NCCN Patient Advocacy Summit, including identified gaps and needs of defining value in cancer care, identified principles and parameters of value tools for patients in cancer care, and consensus statements and recommendations offered by the NCCN Working Group.

\section{Addressing Gaps and Needs of Defining Value From the Patient Perspective}

Gaps between the understanding of value for patients compared with providers and the health system is a central barrier to patient-centered care. ${ }^{1}$ As patients progress through their individual care experience, values, preferences, needs, and treatment options may shift. In order to achieve high-value care, it is imperative that value is defined around the patient. ${ }^{2}$ Moreover, patients need to be empowered with tools that provide health information and education to help them make decisions regarding their individual cancer treatment. Understanding the current gaps and needs of patient-centered care can aid in the future development of value tools for patients.

For a complex disease like cancer, the composition of costs is multifaceted and often shifts throughout the cancer care continuum. Financial toxicity creates an increasing burden on patients. ${ }^{3}$ Furthermore, high costs have both financial and clinical implications for patients. Some patients might expect that their insurance will cover all or most costs associated with their cancer treatment. ${ }^{4}$ Even for insured patients, however, the rising costs of cancer care are increasingly being shifted to patients through premium increases, high deductibles, and copays. More specifically, increases in high deductible plans through the marketplace exchanges, high drug copays including specialty drugs, and a limited network of specialty cancer providers are placing a greater cost burden on patients with cancer. ${ }^{5}$ Premiums and out-of-pocket healthcare costs for a family are projected to equal half of the median household income by 2018 and the entire income by $2040 .^{6}$ Data show that these expenses can affect medication adherence, with $30 \%$ of patients not filling prescriptions and $20 \%$ taking less medication than prescribed due to cost. ${ }^{7}$ Additionally, data suggest that patients who are already financially stretched thin at diagnosis tend to have worse overall health, more pain, greater physical impairment, and deeper depression than those with more savings. ${ }^{8}$ Despite all the identified barriers of financial costs associated with cancer care, only $22 \%$ of patients reported that they recall having cost conversations with their doctor before treatment initiation. ${ }^{9}$ Although individual needs may vary, it is important for patients to manage the myriad financial barriers associated with cancer care so that costs do not become a distraction or an obstruction to their treatment.

With healthcare reform, there is a call for a shift to new value-based payment models; in this vein, value is often understood to be the balance of the benefit of treatment and/or quality of care weighed against economic costs. ${ }^{2}$ As a result, value conversations between patients and providers often inject cost as the most important variable of the equation, potentially overlooking other significant considerations from the patient perceptive ${ }^{4}$; findings from Cancer Support Community's Cancer Experience Registry found that nearly $40 \%$ of surveyed women with metastatic breast cancer defined value in terms of a "personal value," such as QOL, whereas only 7.4\% defined value as an "exchange value," such as cost. ${ }^{10}$ From the patient perspective, high-value care may include trust and transparency with providers, care coordination, survivorship care, QOL, mitigated disparities, toxicity management, and limited travel for care in addition to limited financial risk. Therefore, an essential challenge to address when developing value tools for patients is to understand that patient care is highly individualized and that patient preferences differ and may change over their cancer journey.

Given the multidisciplinary approach to cancer care, it is highly likely that a patient will transition 
Winckworth-Prejsnar et al

across multiple providers during their course of treatment. However, because many electronic health record (EHR) systems lack interoperability, leading to silos within and across healthcare systems, ${ }^{11}$ this creates a major barrier to the implementation of value tools for patients throughout their treatment. When defining value relative to healthcare, patients emphasize the importance of their relationship with providers, rather than just the benefit of cost-effective treatment. ${ }^{12}$ Patients value quality time, interaction, and communication with their clinical team; however, with increasing EHR demands under value-based payment models, reporting burdens for providers can lead to less time for meaningful patient-provider engagement and conversations. Additionally, patients also seek meaningful conversations to understand their treatment options and exert their preferences in terms of QOL and health outcomes. ${ }^{4}$ In other words, patients value conversations to better understand the benefit/risk scenario of their disease and gain confidence that they are making the best treatment decisions. ${ }^{13}$ Recent survey data from Cancer Support Community supports these identified gaps, finding that a quarter of the 1,046 patients surveyed did not feel confident they received the care that they needed, with more than a third of respondents indicating they would have liked to have been more involved in decisions about their care and treatment options. ${ }^{9}$ Overall, patients value cancer care that is highly personalized and customizable to their circumstances; value tools for patients need to account for differences in perceptions around QOL.

Variances in health literacy can contribute to health disparities and increased costs and can impact health outcomes. ${ }^{14}$ Related to the importance of patient-physician communication, health literacy requires communication skills, thoughtful preparation in advance, and parallel lines of communication calibrated for different users. ${ }^{4}$ For some patients, especially those newly diagnosed, caregivers and family members can play a central role in treatment decisions. Panelists highlighted the importance of mechanisms within value tools to allow for meaningful engagement among patients, caregivers, family, and providers.

There is a considerable amount of research from the patient advocate community, collected through surveys and market research, to articulate what patients value and areas where barriers persist. ${ }^{4}$ Overall, however, panelists identified the lack of a comprehensive picture of what patients and families value in terms of the treatment experience, especially as it informs policy around value ${ }^{13}$; the current quality measurement paradigm does not fully capture the value perspective of patients. ${ }^{4}$ Moving forward, it is important to develop measurement capacity in a value tool framework that is sensitive to cost, distress, and the individual existential experience of the cancer care journey, thus informing and shaping high-quality cancer care. Lastly, shared decision-making between providers and patients is not systemic nor is it currently designed to measure whether health outcomes match what patients and families desire to accomplish. ${ }^{4}$ To engage patients to become active partners in their cancer treatment, it is imperative to start with what patients value in order to design value tools for patients that result in optimal value-based care.

\section{Findings and Recommendations for Principles, Parameters, and Measurement of Utility for Value Tools for Patients in Oncology}

In assessing value tools for patients with cancer, the working group noted the varying definitions of "value" and "tool" and developed a common definition of a value tool for patients (Table 1). Using this definition, the group assessed the landscape of current value tools and determined that there is no single tool that comprehensively informs the shared decisionmaking process, guides care planning development, and helps a provider understand patients' unique characteristics and preferences to help provide guidance and determine the best treatment options. Ideally, a comprehensive tool would balance patients' identified needs and allow providers to make more informed recommendations via meaningful shared decision-making conversations. Furthermore, such tools must be patient friendly and easy for a provider to interpret and incorporate into their care work-

\section{Table 1. Working Group Recommendations: Definition of Value Tools for Patients}

A value tool for patients is a dynamic system, process, or device that assists patients in articulating their personal preferences and goals with regard to their medical condition, treatment, and related decision-making. These tools assist patients in communicating information to their providers, which in turn assists providers in delivering personalized care. 
flow; such tools should inform patients, aid care, and not confuse patients or introduce new barriers or administrative burdens.

The working group further identified the need for tools to be dynamic and capture patient values and preferences against evolving treatment options and guidelines. Value tools should enable an iterative process of identifying and addressing patient needs throughout the treatment journey because their values at initial diagnosis may change, which may shift their needs and treatment options. In turn, these tools should allow for meaningful engagement with providers throughout the care journey and also track whether the treatment plan aligns with the patient's values and preferences over time. Ideally, over time, longitudinal patient-centric outcomes at the population level should also be measurable. However, because there is no standardization of such tools in use currently, the working group sought to identify principles and parameters that should be common across value tools, uniform ways of measuring tool utility, and a way to house such tools in a common location, such as a digital library.

Before the summit, the NCCN Working Group identified the principles that would constitute the ideal value tool and the parameters across which to assess their utility (ie, whether the tools are capturing the information intended and leading to more effective and satisfactory patient decision-making). The top 6 principles were ranked by the working group members in domains of importance, developmental feasibility, and implementation feasibility (Table 2).

To establish a uniform way of measuring value tool utility, the working group proposed several questions to evaluate the utility of future tools (Table 3 ). The group determined that value measurement is not a single-point-in-time activity; feedback loops must be in place at the provider-patient level, when the tool is in use, and at the macro level with the tool developer, so that it may be refined, improved, and updated to reflect relevant evidence-based clinical guidelines over time. The working group also recommended the future development of a library of value tools for patients to help increase knowledge of existing tools, and to collect and establish longitudinal data for tool utility and patient outcomes measurement.

Building off of the NCCN Working Groupidentified recommendations, further discussion at the summit led to additional principles and param- eters outlined by panelists (Table 4). Panelists reiterated that just as cancer is a heterogeneous disease, there also exists a heterogeneity of patient preferences throughout the cancer care continuum. ${ }^{13}$ Certain domains, such as cost, efficacy, and side effects, may have different weight to a patient initially, but may change over time or be replaced by other factors they deem more important, such as overall survival or QOL. Therefore, as discussed previously, it is paramount that value tools are dynamic to patients' changing preferences as their disease evolves. In order to truly measure utility, data from value tools must be extractable and shareable across providers. Given the multidisciplinary nature of cancer care, the best patient experience is a seamless one in which all providers on the care team have all of the same patient knowledge, whether it is values and preferences or past diagnostics and treatments. Therefore, panelists recommended that, regardless of each tool's platform, data must be meaningfully integrated into an EHR, interoperable with other provider systems, and exportable for population health management and outcomes research. Thus, all of the information is housed in one place for the care team and the patient (if the EHR has a patient portal).

In the short term, value tools for patients should encompass the relevant principles and parameters identified by the working group and panelists and be validated for their utility using the relevant questions identified by the working group (Table 3). In the long term, value tools should be (1) standardized for population-based measurement and ease of clinician judgment and use; (2) scalable and customizable to account for individual patient factors (eg, preferences and place on care journey) that may change over time; (3) visible and usable across the care continuum by multidisciplinary providers involved in a patient's care; (4) mutually interactive by both patients and providers; and (5) available in a library accessible for patients and providers.

\section{Value Tools for Patients: The Path Forward}

During the summit, Dr. Sandhya Pruthi from the Mayo Clinic demonstrated a Web-based tool accessible on a mobile device that addressed a number of identified working group recommendations. In 2015, the Breast Cancer Surgical Decision Support iPad 
Winckworth-Prejsnar et al

\begin{tabular}{|c|c|c|c|}
\hline Rank & Importance & Development Feasibility & Implementation Feasibility \\
\hline 1 & Captures patient preferences and values & Understandable language & User-friendly \\
\hline 2 & $\begin{array}{l}\text { Support care workflow, including } \\
\text { feedback loop }\end{array}$ & User-friendly & Understandable language \\
\hline 3 & $\begin{array}{l}\text { Ability to personalize tool to patients' } \\
\text { needs/place on treatment journey }\end{array}$ & Captures patient preferences and values & $\begin{array}{l}\text { Captures patient preferences and } \\
\text { values }\end{array}$ \\
\hline 4 & Support patients' individual life flow & Pretool needs assessment screening & $\begin{array}{l}\text { Ability to personalize tool to patients' } \\
\text { needs/place on treatment journey }\end{array}$ \\
\hline 5 & Understandable language & Allows room for clinical judgment & Allows room for clinical judgment \\
\hline 6 & User-friendly & Support patients' individual life flow & $\begin{array}{l}\text { Support care workflow including } \\
\text { feedback loop }\end{array}$ \\
\hline \multicolumn{4}{|c|}{ Additional principles and parameters not ranked } \\
\hline \multicolumn{4}{|c|}{ - Exportability to different electronic health records and technology environments } \\
\hline \multicolumn{4}{|c|}{ - Scalability of tool across the continuum of care } \\
\hline \multicolumn{4}{|c|}{ - Ability to report patient-reported outcomes data } \\
\hline \multicolumn{4}{|c|}{ - Ability to track patient value and preference changes over time } \\
\hline
\end{tabular}

Tool was introduced as an educational tool for patients at the Mayo Clinic. Patients were loaned an iPad with interactive and personalized breast cancer information about surgical, medical, radiation, and reconstructive options available during their preoperative journey. More specifically, this tool included information about risks, benefits, timelines, and recovery, with an interactive component to assess patient values, confidence, and readiness for surgery, with a feedback loop to a provider-facing dashboard

\section{Table 3. Working Group Recommendations:} Questions for Utility

- Did shared decision-making occur after using the tool?

- Were patients satisfied with their care experience?

- Were pros and cons of alternatives discussed?

- Were patient preferences and goals accounted for?

- Did the patient have a chance to explain their understanding of the situation and available options?

- How happy was the patient with his/her care choice after the fact?

- What patient-reported outcomes were achieved?

- Did the care plan work for the patient?

- Was the outcome sensitive to the patient's preferences (eg, long-term survival not always the patient's desired end point)?

- End points may include: survivorship, quality of life, and specific symptom management or avoidance

- Was there a trade-off between patient satisfaction and clinical quality of care (eg, narcotic/antibiotic prescriptions, sensitive conversations regarding smoking, drugs, and weight)?

- Are outcomes accessible short-term and trackable long-term?

- Did the tool work for measuring patient satisfaction and experience, and did it fit into clinical workflow so providers could incorporate patient values and preferences and care delivery? accessible by the multidisciplinary breast specialists and care team. Dr. Pruthi reported high patient satisfaction with the Web-based tool and improved confidence in surgical decision-making among patients. ${ }^{15}$

A patient who used the Web-based tool described the anxiety-provoking experience of receiving her cancer diagnosis; she was scared, overwhelmed, and had a difficult time retaining any important information about her diagnosis. ${ }^{13}$ She explained that the educational tool enabled easy access to the content and information, was easy to use and navigate through to the next steps in her treatment, and was invaluable for her and her family members. Moreover, the tool provided the necessary educational content using an algorithmic format to help her feel confident in her surgery decision, which she described as a greater sense of empowerment. Patients can search the internet for an overwhelming amount of resources that might include various types of breast cancer and prognosis information, ${ }^{13}$ but this tool provided access to information in one central repository with immediate availability and utility; her only suggestion was that the tool be readily accessible at diagnosis. Dr. Pruthi and colleagues will continue to evaluate the impact of a cancer educational tool using mobile technology to enhance the patient experience and quality of breast cancer care. Moving forward, Dr. Pruthi highlighted and identified a need to expand the tool into survivorship care and to allow personalization and interactivity to be built into these tools for the entire journey of a patient's care continuum. ${ }^{13}$ 
Value Tools for Patients

\section{Table 4. Panelist Recommendations: Principals and Parameters \\ - Allow for immediacy and timeliness of information to the patient and of utility to the provider \\ - Enable interactive and iterative dialogue between patient and provider \\ - Account for differences in each patient's quality of life values \\ - Be intuitive so that extensive training on the part of patient or provider is not required \\ - Empower patient's to identify their values and raise questions back to the provider \\ - Offer tool through multiple modalities (eg, via paper, phone, computer, community health workers, health navigators, public libraries) \\ - Be sensitive to varying health literacy levels and language \\ - Allow patient values to be revisited and adaptable over time \\ - Include cost conversations beyond drug and treatment costs (eg, transportation, emergency room visits due to side effects)}

\section{Conclusions}

The oncology community is moving toward a greater emphasis on individual patient preferences and values shaping multiple aspects of the healthcare system as a key strategy for achieving patient-centered care delivery. Thus, a health system where consumer engagement will have the most impact is one built on the principles of patient-centered care. Although the concept of patient-centered care is often discussed in great detail, the oncology commu- nity has not fully systemized the philosophical idea of the value of a patients' preferences and those that shape the cancer care journey. ${ }^{16}$ Moreover, in an area of scarce resources, it will continue to be important to allocate resources to best match what a patient values. Ultimately, there is an identified need to optimize patient value in a way that is still responsible and cognizant of societal limits in resource allocation. It behooves all oncology stakeholders to start collecting broader data sets, beyond traditional clinical trial data (eg, overall survival, progressionfree survival, anemia, transfusions, hospitalizations), to accelerate a comprehensive conversation around value from the patient perspective. ${ }^{13}$ Lastly, with the shift from volume to value within the alternative payment model environment, there is a window of opportunity to not only think about how to pay for care differently, but also how to deliver it differently based on the development of value tools for patients. As the oncology community continues to develop value tools for patients that incorporate personalized information around costs, benefits, and risks to patients, in conjunction with appropriate feedback loop mechanisms informing the shared decisionmaking and care planning process, the system can achieve optimal patient-centered care delivery.

\section{References}

1. NCCN Policy Summit. Value, Access, and Cost of Cancer Care; September 11, 2015; Washington DC.

2. Porter ME. What is value in health care? N Engl J Med 2010;363:24772481

3. Zafar SY, Abernethy AP. Financial toxicity, part I: a new name for a growing problem. Oncology (Williston Park) 2013;27:80-81, 149

4. Gaps of value tools for patients in cancer care. Panel discussion at the NCCN Patient Advocacy Summit: Value Tools for Patients; December 9 , 2016; Washington, DC.

5. American Society of Clinical Oncology. The state of cancer care in America, 2017: a report by the American Society of Clinical Oncology. J Oncol Pract 2017;13:e353-394.

6. Young RA, DeVoe JE. Who will have health insurance in the future? An updated projection. Ann Fam Med 2012;10:156-162.

7. Zafar Y, Goetzinger AM, Fowler R, et al. Impact of out-of-pocket expenses on cancer care [abstract]. J Clin Oncol 2011;29(Suppl):Abstract 6006

8. Lathan C, Cronin A, Tucker-Seeley R, et al. Association of financial strain with symptom burden and quality of life for patients with lung or colorectal cancer. J Clin Oncol 2016;34:1732-1740.

9. Cancer Support Community. Access to Care in Cancer 2016: Barriers and Challenges. Available at: http://www.cancersupportcommunity.org/ sites/default/files/uploads/policy-and-advocacy/patient-access/access_to_

care_in_cancer_2016_barriers_and_challenges_slide_deck.pdf. Accessed February 21, 2017.

10. Longacre ML, Charap ES, Buzaglo JS, et al. Defining value in oncology: perspectives from patients with metastatic breast cancer. Presented at the Association for Value-Based Cancer Care 5th Annual Conference; May 3-6, 2015; Washington, DC.

11. Nardi EA, Lentz LK, Winckworth-Prejsnar K, et al. Emerging issues and opportunities in health information technology. J Natl Compr Canc Netw 2016;14:1226-1233.

12. House L. Cancer experience registry. Presented at the NCCN Value Tools for Patients in Cancer Care Working Group Meeting; August 25, 2016; Philadelphia, PA.

13. Understanding principles and parameters of value tools for patients in oncology. Panel discussion at the NCCN Patient Advocacy Summit: Value Tools for Patients; December 9, 2016; Washington, DC.

14. Morris NS, Field TS, Wagner JL, et al. The association between health literacy and cancer-related attitudes, behaviors, and knowledge. J Health Commun 2013;18(Suppl 1):223-241.

15. Pruthi S. Demonstration of value tool for patients: breast cancer surgical decision support iPad tool. Presented at the NCCN Patient Advocacy Summit: Value Tools for Patients; December 9, 2016; Washington, DC.

16. Balch A. Findings and recommendations of NCCN value tools for patients working group. Presented at the NCCN Patient Advocacy Summit: Value Tools for Patients; December 9, 2016; Washington, DC. 